Original Research Paper

\title{
Highlight of New Phosphodiesterase 10A Inhibitors Using Molecular Docking
}

\author{
Halla Belhoula*, El Hassen Mokrani, Abderrahmane Bensegueni and Djihane Bioud \\ Department of Biochemistry and BCM, Faculty of Nature and Life Sciences, Laboratory of Applied Biochemistry, \\ University brother Mentouri Constantine 1, USA
}

Article history

Received: 25-12-2019

Revised: $13-01-2020$

Accepted: 24-02-2020

Corresponding Author:

Halla Belhoula

Department of Biochemistry and BCM, Faculty of Nature and Life Sciences, Laboratory of Applied Biochemistry, University brother Mentouri Constantine 1, USA

Email: maya20fao@hotmail.com

\section{Introduction}

Many countries today have mental illness as a major public health issue. Among these diseases, Schizophrenia (SCZ) is the leading cause of severe and prolonged disability. In fact, in some countries, SCZ currently occupy more than half of psychiatric hospital beds. Since SCZ begins early in adulthood and does not significantly reduce life expectancy, not only are these patients removed from society to a period that should be that of their highest productivity. However, since they are often in good physical condition, their stay in hospital can last for very long years. It is therefore of the utmost importance to make every effort to prevent and treat this disease (Suzuki and Kimura, 2018). The main drugs used to treat symptoms of SCZ are antipsychotics. Although they are useful to most people with SCZ, antipsychotics can have serious side effects, let us take as examples: Motion disturbance, dizziness, cardiac arrhythmia, weight gain that increases the risk of diabetes and cardiovascular disease (Geddes et al., 2000). Among the various treatments of SCZ, Phosphodiesterase 10A (PDE 10A) inhibition appeared as a new and very effective therapeutic approach. PDE 10A inhibitors represent a new treatment for the negative, positive and the cognitive symptoms of SCZ, with a lower risk of side effects than conventional antipsychotics (Wagner et al., 2015).

Molecular docking is one of the most used computational methods in structure based drug design. It comprise two distinct tasks; the first being the prediction of favorable binding geometries for a small molecule in the binding site of a target protein and secondly, the estimation of the binding free energy of the complex so formed, also referred to as scoring (Kitchen and Decornez, 2004; Halperin et al., 2002).

The purpose of this work is to highlight new potent PDE 10A inhibitors using molecular docking method.

\section{Materials and Methods}

\section{Target Preparation}

The 3D structure of the PDE 10A; subject of our study, has been acquired from Protein Data Bank under the code 5UWF. This code corresponds to the $3 \mathrm{D}$ structure of the PDE $10 \mathrm{~A}$ in complex with a cocrystallized inhibitor that has the code 8Q7. PDE 10A is a homodimeric enzyme; we have therefore eliminated one of its chain. This last was separated from its inhibitor (8Q7) in order to keep only the enzyme with free active site. Then, water molecules were also eliminated Afterwards, all the missing hydrogens are added and the partial loads of type "Kollman" are calculated with the Autodock Tools program. The prepared protein is stored in a pdbqt format file.

\section{Ligand Preparation}

The 3D structures of the PDE 10A inhibitors were drawn, minimized and exported as pdb files using Titan program. Then, all hydrogen atoms were added using Autodock Tools. This last program was also used in order to define aromatic carbons and rotatable bonds of each compound. The prepared ligands were exported as $p d b q t$ files. 


\section{Docking Calculations}

Molecular docking calculations were undertaken using the last version of AutoDock (Morris et al., 1998). The compound $\mathrm{a}$ is one of the best PDE $10 \mathrm{~A}$ inhibitors (Tuttle and Kormos, 2014) with a value of $\mathrm{IC}_{50}$ of $0.4 \mathrm{nM}$. This compound was taken as starting structure for search of similar compounds. In this context, 396 similar compounds of a were downloaded from PubChem Database and prepared for docking calculations against active site of PDE 10A. According to docking's result, the structure of best similar compound was modified in order to define a new potent PDE $10 \mathrm{~A}$ inhibitor.

\section{Assessment of Docking Quality}

The RMSD test represents the ability of a program to reproduce the experimental binding modes of a ligand. The RMSD value is equal to the average of the deviation of each of atoms compared to the original molecules. The best value of mean RMSD between the placing of the ligand calculated AutoDock and the conformation in the experimental complex was the smallest possible. The ratio accepted is $2 \AA$ beyond which the prediction is considered irrelevant. The current standars for evaluating the performance of a docking program is to make a test from hundreds of protein-ligand complexes crystallized. This test was performed on 100 complexes available in the PDB and the RMSD determined.

\section{Drug Likeness Prediction}

The pharmacokinetic parameters consisting of BloodBrain Barrier (BBB) penetration, gastrointestinal absorption (GI), Cytochrome P450 (CYP) inhibition and Lipinski's rule of 5 of the best compound found in this word were predicted using Suissadme at http://www.swissadme.ch/index.php. Its toxicity was predicted using. PreADMET at https://preadmet.bmdrc.kr.

\section{Results and Discussion}

\section{Validation of Docking Protocol}

The performance of AutoDock was evaluated using 100 complexes Protein-Ligand available in the PDB. For each complex, the predicted binding mode was compared with the experimental binding conformation and a Root Mean Square Distance (RMSD) between the two models was calculated by AutoDock. A prediction of a binding mode was considered successful if the RMSD was below $2 \AA$ (Gabb et al., 1997). As shown in Fig. 1, the program AutoDock reproduced well the experimental data. Indeed, $71 \%$ of RMSD values are less than $2 \AA$.

\section{Virtual Screening of Similar Compounds Collection}

The compound $\mathbf{a}$ is one of the best PDE 10A inhibitors (Tuttle and Kormos, 2014). Its experimental $\mathrm{IC}_{50}$ value is $0.4 \mathrm{nM}$. The binding energy between this compound and PDE 10A active site was predicted by molecular docking. The obtained value of binding energy $(-10.28 \mathrm{Kcal} / \mathrm{mol})$ showed a satisfactory agreement with the experimental data. In order to find new potent PDE 10A inhibitors, 369 similar compounds of a from PubChem database were docked into the active site of PDE 10A. It appears that the compound S235 was predicted as more potent PDE $10 \mathrm{~A}$ inhibitor than the starting compound (a) since its binding was improved from $-10.28 \mathrm{Kcal} / \mathrm{mol}$ to $-11.95 \mathrm{Kcal} / \mathrm{mol}$.

The binding mode of S235 into the active site of PDE10A was analyzed using the Maestro program. Figure 2 showed that S235 establish a hydrogen bond with Leu675.

\section{Structural Modification of S235}

In order to design new potent PDE10A inhibitor, the structure of S235 was modified. In this context, the cyclohexane ring of $\mathrm{S} 235$ was replaced by 1,4,5,6tetrahydropyrimidine and its piperidine by a piperazine ring. The binding mode of the resulted compound, called a1, was predicted. Remarkably, the binding energy of this compound was improved from $-11.95 \mathrm{Kcal} / \mathrm{mol}$ (S235) to $-13.80 \mathrm{Kcal} / \mathrm{mol}$. It should be noted that the improvement in the predicted binding energy is due to the setting up of new links and interactions because of the structural modifications performed. As shown in Fig. 3, al forms six hydrogen bonds with amino acids residues of PDE $10 \mathrm{~A}$ active site.

Table1: The predicted pharmacokinetic and toxicity parameters of compound al

\begin{tabular}{ll}
\hline Properties & Compound a1 \\
\hline $\mathrm{BBB}^{\mathrm{a}}$ & Yes \\
$\mathrm{GI}^{\mathrm{b}}$ & High \\
$\mathrm{CYP}^{\mathrm{c}}$ inhibitor & No \\
Lipinski's rule of 5 & Suitable \\
Toxicity & $\mathrm{CR}^{\mathrm{d}}$ \\
& $\mathrm{hERG}^{\mathrm{e}}$ inhibition \\
\hline
\end{tabular}

${ }^{a}$ BBB: Blood-Brain Barrier. ${ }^{b}$ GI: Gastrointestinal absorption. ${ }^{\mathrm{c} C Y P}$ : Cytochrome P450. ${ }^{\mathrm{d}} \mathrm{CR}$ : Carcinogenicity in Rat. ${ }^{\mathrm{e}} \mathrm{hERG}$ : Human Ether-a-go-go Related Gene channel

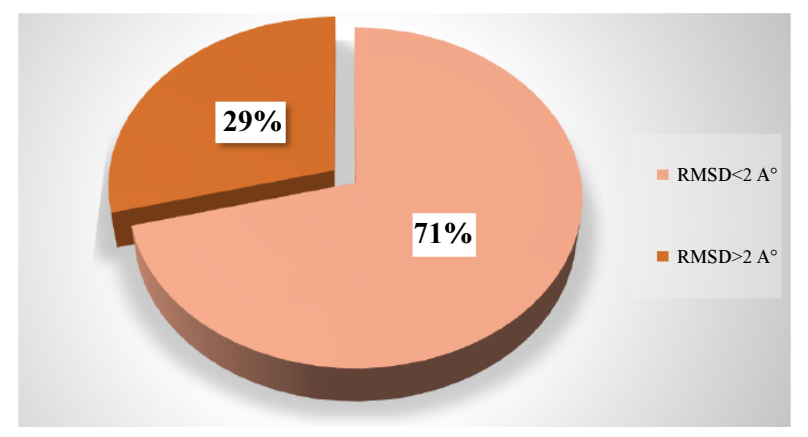

Fig. 1: Results in\% obtained by AutoDock at two intervals of $\operatorname{RMSD}(\AA)$ 


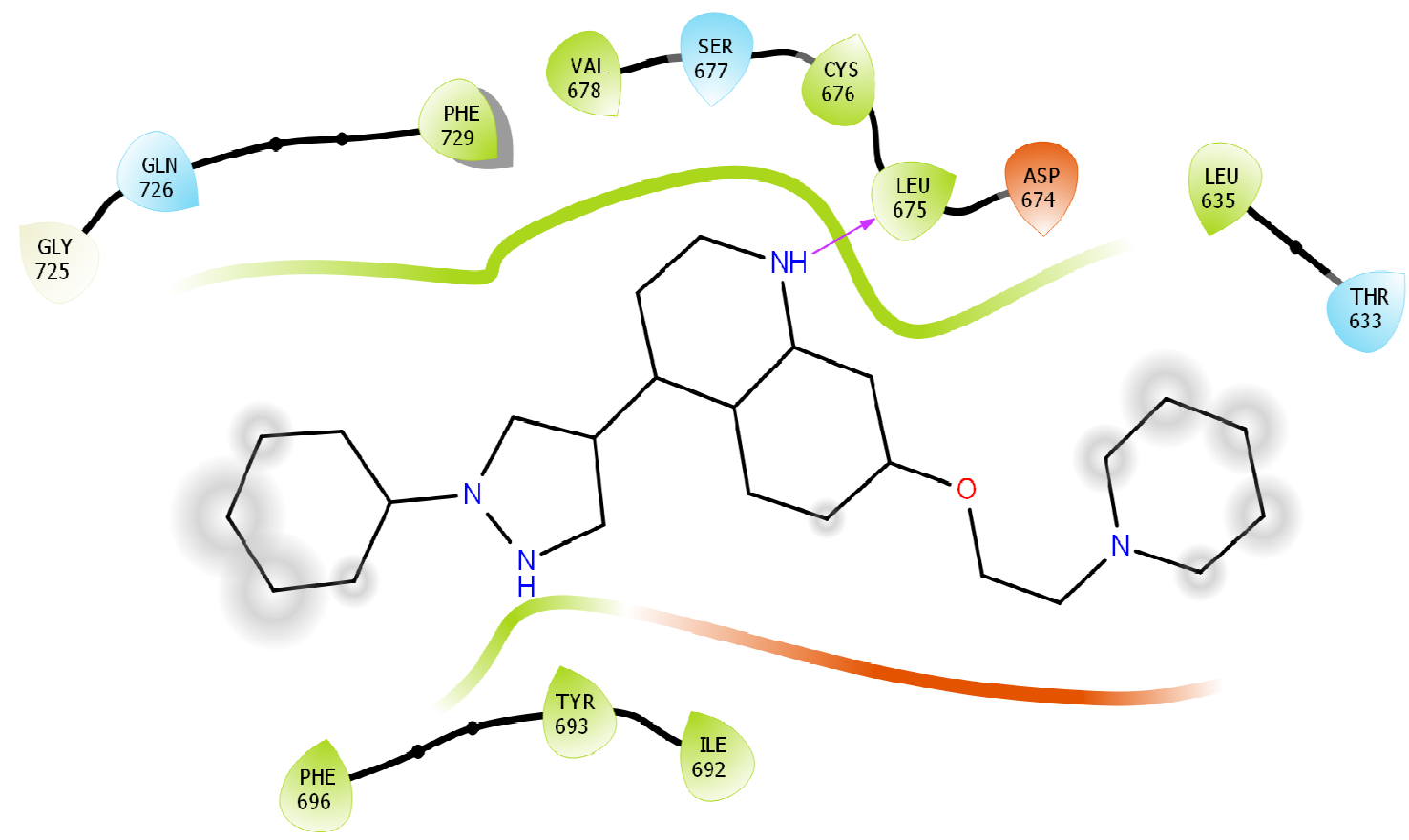

Fig. 2: Binding mode prediction of S235 into the PDE10A active pocket. Purple arrowhead from the donor to the acceptor of hydrogen bond. The images were done with the ligand interaction diagram script from the Schrçdinger suite

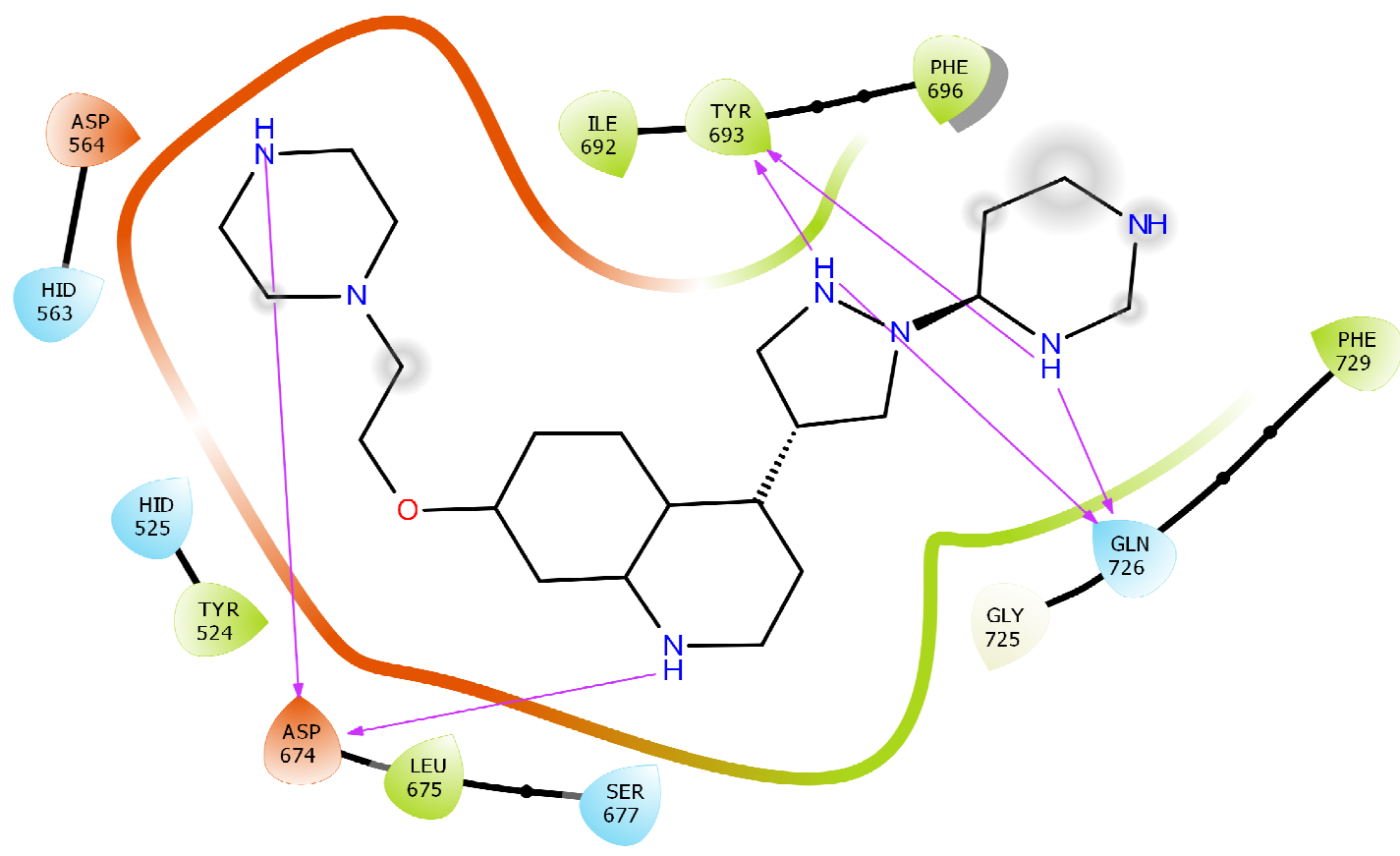

Fig. 3: Binding mode prediction of al into the PDE10A active pocket. Purple arrowhead from the donor to the acceptor of hydrogen bond. The images were done with the ligand interaction diagram script from the Schrçdinger suite

\section{Drug Likeness Prediction}

Finally, we predicted the pharmacokinetic and toxicity parameters of the most promising PDE10A inhibitor. As shown in Table 1, a1 had a high possibility in gastrointestinal absorption. Furthermore, this compound was not predicted to inhibit CYP (enzymes that should not be inhibited because they are essential for 
the metabolism of many drugs in the liver). With no Lipinski's rule of 5 violation, al follows the criteria for orally available drugs. However, his toxicity problems including $\mathrm{CR}$ and hERG inhibition can be improved during its optimization.

\section{Conclusion}

In bref, this study showed that the AutoDock program can be used to predict enzyme-inhibitors interactions.

The strategy undertaken in this work, consisting of the docking of 369 compounds followed by a series of structural modifications allowed as identify a new potent PDE10A inhibitor. The binding mode of the most promising inhibitor was analyzed and showed that this compound cover the binding site of PDE10A in a rational orientation, where their hydrogen bonds with TYR693, GLN726, ASP674 seems to play an important role, leading to its high inhibitory potency. This new compound possess satisfying drug-like properties, indicating that it might be promising lead compound for further research.

\section{Acknowledgment}

The authors gratefully thank the Laboratory of Applied Biochemistry, Department of Biochemistry and BCM, Faculty of Nature and Life Sciences, University brother Mentouri Constantine 1.

\section{Author's Contributions}

The first, the second and the last authors were participated in all experiments, coordinated the dataanalysis and contributed to the writing of the manuscript, while the third author revised and modified the manuscript.

\section{Ethics}

This research was subjected to ethical clearance from the authrorities of University brother Mentouri Constantine 1, Algeria.

\section{References}

Gabb, J., R.M. Jackson and M.J.E. Sternberg, 1997. Modelling protein docking using shape complementarity, electrostatics and biochemical information. J. Molecular Biol., 272: 106-120. DOI: $10.1006 /$ jmbi.1997.1203

Geddes, J., N. Freementale, P. Harrisson and P. Bebbington, 2000. Atypical antipsychotics in the treatment of schizophrenia: Systematic overview and meta-regression analysis. British Med. J., 321: 1371-1376. DOI: 10.1136/bmj.321.7273.1371
Halperin, I., B. Ma and H. Wolfson, 2002. Principles of docking: An overview of search algorithms and a guide to scoring functions. Proteins, 4: 409-430. DOI: $10.1002 /$ prot. 10115

Kitchen, D.B. and H. Decornez, 2004. Docking and scoring in virtual screening for drug discovery: Methods and applications. Nature Rev. Drug Dis., 3 : 935-949. DOI: 10.1038/nrd1549

Morris, G.M., D.S. Goodsell, R.S. Halliday, R. Huey and W.E. Hart et al., 1998. Automated docking using a Amarckian genetic algorithm and an empirical binding free energy function. J. Comput. Chem., 19: 1639-1662.

DOI: 10.1002/(SICI)1096987X(19981115)19:14<1639::A ID-JCC $10>3.0 . \mathrm{CO} ; 2-\mathrm{B}$

Suzuki, K. and H. Kimura, 2018. TAK-063, a novel PDE10A inhibitor with balanced activation of direct and indirect pathways, provides a unique opportunity for the treatment of schizophrenia. CNS Neurosci. Therapeut., 7: 604-614.

DOI: $10.1111 / \mathrm{cns} .12798$

Tuttle, J.B. and B.L. Kormos, 2014. The use of PDE10A and PDE9 inhibitors for treating schizophrenia. Topics Med. Chem., 13: 255-316. DOI: 10.1007/7355_2014_54

Wagner, S., M. Scheunemann, K. Dipper, U. Egerland and N. Hoefgen et al., 2015. Development of highly potent phosphodiesterase 10A (PDE10A) inhibitors: Synthesis and in vitro evaluation of 1,8-dipyridinyland 1- pyridinyl-substituted imidazo[1,5a]quinoxalines. Eur. J. Med. Chem., 107: 97-108. DOI: 10.1016/j.ejmech.2015.10.028

\section{Abbreviation and Units}

$\begin{array}{ll}3 \mathrm{D} & \text { Three Dimensions } \\ \AA & \text { Angstrom } \\ \text { ADMET } & \begin{array}{l}\text { Absorption Distribution } \\ \text { Excretion Toxicity }\end{array} \\ & \text { 50\% Inhibitory Concentration } \\ \mathrm{IC}_{50} & \text { Kilo calories/moL } \\ \mathrm{KcaL} / \mathrm{moL} & \text { nanomolar } \\ \mathrm{nM} & \text { Protein Data Bank } \\ \mathrm{PDB} & \text { Phosphodiesterase 10A } \\ \text { PDE 10A } & \text { Root Mean Square Deviation } \\ \text { RMSD } & \text { Schizophrenia } \\ \text { SCZ } & \end{array}$

